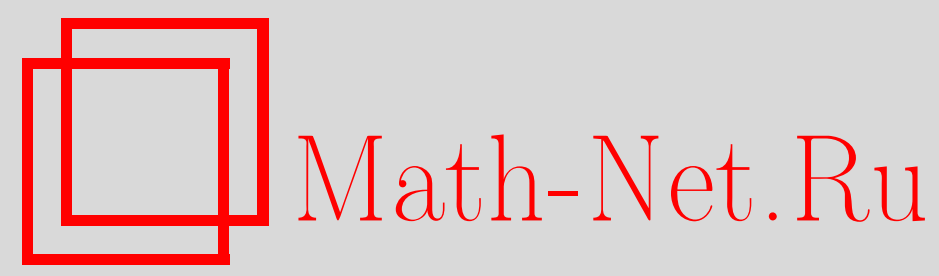

А. Н. Тимашев, Об асимптотических разложениях в области больших уклонений для биномиального и пуассоновского распределений, Теория вероятн. и ее примен., 1998, том 43, выпуск 1, 57-68

DOI: https://doi.org/10.4213/tvp823

Использование Общероссийского математического портала Math-Net.Ru подразумевает, что вы прочитали и согласны с пользовательским соглашением http://www . mathnet.ru/rus/agreement

Параметры загрузки:

IP : 54.89 .56 .158

26 апреля 2023 г., $17: 40: 24$

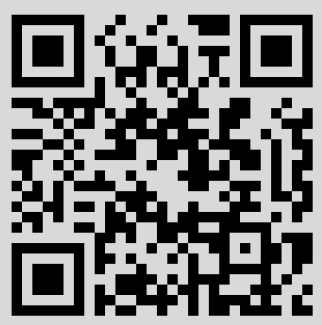




\section{ОБ АСИМПТОТИЧЕСКИХ РАЗЛОЖЕНИЯХ В ОБЛАСТИ БОЛЬШІИХ УКЛОНЕНИЙ ДЛЯ БИНОМИАЛЬНОГО И ПУАССОНОВСКОГО РАСПРЕДЕЛЕНИЙ}

Рассматривается случайная величина $\xi$, распределенная по биномиальному закону с параметрами $n$ и $p(0<p<1)$. Решается задача асимптотической оценки при $n \rightarrow \infty$ и постоянном $p$ вероятности $\mathbf{P}\{\xi \geqslant k\}$ при условии, что $k \rightarrow \infty(k \in \mathbf{N})$ так, что $p<\alpha_{0} \leqslant \alpha=k / n \leqslant \alpha_{1}<1\left(\alpha_{0}, \alpha_{1}\right.$ - постоянные).

Далее рассматривается случайная величина $\eta$, распределенная по закону Пуассона с параметром $\lambda>0$. При $\lambda \rightarrow+\infty$ находятся асимптотические оценки вероятности $\mathbf{P}\{\eta \geqslant k\}$ при условии, что $k \rightarrow$ $\infty$ так, что $k \in \mathbf{N} ; 1<\gamma_{0} \leqslant \gamma=k / \lambda \leqslant \gamma_{1}\left(\gamma_{0}, \gamma_{1}\right.$ - постоянные).

Методом перевала получены разложения указанных вероятностей в асимптотические степенные ряды по степеням переменных $n^{-1}$ и $\lambda^{-1}$, коэффициенты которых удовлетворяют некоторым линейным рекуррентным соотношениям в комплексной области с заданными начальными условиями.

Ключевые слова и фразы: биномиальное распределение, распределение Пуассона, асимптотическое разложение, метод перевала.

Пусть $\xi$ - случайная величина, распределенная по биномиальному закону с параметрами $n$ и $p(0<p<1)$, т.е. $\xi \sim \mathrm{B}(n, p)$. Рассматривается задача асимптотической ощенки при $n \rightarrow \infty$ вероятности $\mathbf{P}\{\xi \geqslant k\}$ при условии, что $k \rightarrow \infty(k \in \mathbf{N})$ так, что $p-$ фиксированное число, принадлежашее интервалу $(0,1)$, и

$$
p<\alpha_{0} \leqslant \alpha=\frac{k}{n} \leqslant \alpha_{1}<1
$$

$\left(\alpha_{0}, \alpha_{1}\right.$ - постоянные)

С помошью метода перевала получено разложение этой вероятности в асимптотический степенной ряд по степеням переменной $n^{-1}$, коэффициенты которого удовлетворяют некоторым линейным рекуррентным соотношениям в комплексной области с заданными начальными условиями. Заметим, что оценки «хвоста» биномиального распределения имеются во многих монографиях, учебниках и статьях. При этом обычно ограничиваются точными или асимптотическими оценками главного

*Академия ФСБ, Мичуринский пр., 70, 125040 Москва, Россия. 
члена разложения при условии (1) либо рассматривают пругие области изменения параметров $n$ и $\boldsymbol{p}$ (в частности, область притяжения нормального закона, для которой известно полное асимптотическое разложение). По-видимому, впервые выражение для главного члена асимптотического степенного ряда при условии (1) появилось в [1] (см. также работу автора [2], где этот результат обобщается на случай полиномиального распределения с фиксированными вероятностями исходов и растущим числом испытаний).

Далее рассматривается случайная величина $\eta$, распределенная по закону Пуассона с параметром $\lambda>0$, и оценивается «хвост» пуассоновского распределения, т.е. вероятность $\mathbf{P}\{\eta \geqslant k\}$ при условии, что $k \rightarrow \infty$ при $\lambda \rightarrow+\infty$ и

$$
1<\gamma_{0} \leqslant \gamma=\frac{k}{\lambda} \leqslant \gamma_{1}<+\infty
$$

( $k \in \mathbf{N} ; \gamma_{0}, \gamma_{1}$ - постоянные).

Применяя метод перевала, мы получаем разложение этой вероятности в асимптотический степенной ряд по степеням переменной $\lambda^{-1}$, коэффициенты которого удовлетворяют некоторым линейным рекуррентным соотношениям с заданными начальными условиями.

Для получения искомого асимптотического разложения при условии (1) или (2) в обоих случаях применяется один и тот же метод, позволяющий выписать рекуррентные соотношения для коэффициентов разложения, если величина, которую нужно оценить, может быть представлена в виде контурного интеграла с подынтегральной функцией специального вида. Идея метода имеется в $[3$, с. 66], [4, с. 165] и [5, с. 79]. По-видимому, таким же способом могут быть получены асимптотические разложения и в некоторых других комбинаторно-вероятностных задачах.

Чтобы оценить вероятность $\mathbf{P}\{\xi \geqslant k\}$ при условии (1), будем исходить из равенств

$$
\mathbf{P}\{\xi \geqslant k\}=\frac{1}{2 \pi i} \oint \frac{1-z(q+p z)^{n}}{(1-z) z^{k+1}} d z \quad(k=0,1, \ldots, n) .
$$

Эти равенства следуют из легко проверяемой формулы

$$
\sum_{k=0}^{n} \mathbf{P}\{\xi \geqslant k\} z^{k}=\frac{1-z(q+p z)^{n}}{1-z}
$$

справедливой при $z \neq 1$. При этом полагаем $q=1-p$; интегрирование в этих равенствах осуществляется по окружности радиуса $R$ с центром в нуле, пробегаемой в положительном направлении. Далее будем считать, что $R>1$. Используя теорему о вычетах, нетрудно убедиться в том, что в этом случае

$$
\oint_{|z|=R>1} \frac{d z}{(1-z) z^{k+1}}=0 \quad(k=0,1, \ldots, n) .
$$


Таким образом,

$$
\mathbf{P}\{\xi \geqslant k\}=\frac{1}{2 \pi i} \oint_{|z|=R>1} \varphi(z) \exp (n \omega(z)) d z
$$

где

$$
\varphi(z)=(z-1)^{-1}
$$

И

$$
\omega(z)=\ln (q+p z)-\alpha \ln z
$$

если выполнено условие (1).

Заметим, что в формуле (5) выбирается та непрерывная ветвь логарифма, которая принимает вещественные значения на вещественной положительной полуоси. Кроме того, из уравнения $\omega^{\prime}(z)=0$ следует, что $z=z_{0}=\alpha(1-p) /[p(1-\alpha)]>1$, если справедливо (1). В дальнейшем будем считать, что в формуле (3) $R=z_{0}>1$.

Теорема 1. IIусть прu $n \rightarrow \infty 0<p=$ const $<1 u k \rightarrow \infty$ $(k \in \mathbf{N})$ так, что выполнено условие (1). Тогда равномерно относительно $\alpha \in\left[\alpha_{0}, \alpha_{1}\right]$ справедливо асимптотическое разложение

$$
\begin{aligned}
\mathbf{P}\{\xi \geqslant k\} \backsim & \frac{1-p}{\alpha-p} \sqrt{\frac{\alpha}{2 \pi(1-\alpha) n}} \exp (-n \dot{J}) \\
& \times\left(1+\sum_{m=1}^{\infty} \frac{(2 m) !}{m ! 2^{2 m}} \frac{x_{2 m}}{n^{m}}\right),
\end{aligned}
$$

2de

$$
J=\alpha \ln \frac{\alpha}{p}+(1-\alpha) \ln \frac{1-\alpha}{1-p}>0
$$

$u$ коэффичиенты $x_{m}(m=1,2, \ldots)$ удовлетворяют линейным рекуррентным соотношениям в.комплексной области:

$$
x_{m}=-\frac{i p(1-\alpha)^{3 / 2}}{(1-p) \sqrt{2 \alpha}}(m+1) c_{m+1}-\frac{p(1-\alpha)}{\alpha-p} \sum_{r=0}^{m-1} c_{m-r} x_{r}
$$

$(m=1,2, \ldots)$ с начальным условием $x_{0}=1$.

$B$ формуле (7) коэффичиенты $c_{r}(r=1,2, \ldots)$ удовлетворяют нелинейным рекуррентным соотношениям в комплексной области:

$$
\begin{aligned}
c_{r+1}= & \frac{i}{r+2} \sqrt{\frac{1-\alpha}{2 \alpha}}\left(\frac{2(1+\alpha)}{1-\alpha} c_{r}\right. \\
& \left.+\frac{p}{1-p} \sum_{s=1}^{r-1}\left(2 c_{s} c_{r-s}+(1-\alpha)(s+1) c_{s+1} c_{r-s+1}\right)\right)
\end{aligned}
$$

$(r=2,3, \ldots)$ с начальными условиями

$$
c_{1}=\frac{i(1-p)}{p(1-\alpha)} \sqrt{\frac{2 \alpha}{1-\alpha}} ; \quad c_{2}=-\frac{2(1+\alpha)(1-p)}{3 p(1-\alpha)^{2}} .
$$


Д о к а 3 а т е л ь с т в о. Прежде чем доказывать теорему 1, заметим, что (6) согласно определению асимптотического разложения означает, что при условиях этой теоремы для любого фиксированного значения $M=1,2, \ldots$ равномерно относительно $\alpha \in\left[\alpha_{0}, \alpha_{1}\right]$ справедливо равенство

$$
\begin{aligned}
\mathbf{P}\{\xi \geqslant k\}= & \frac{1-p}{\alpha-p} \sqrt{\frac{\alpha}{2 \pi(1-\alpha) n}} \exp (-n J) \\
& \times\left(1+\sum_{m=1}^{M} \frac{(2 m) !}{m ! 2^{2 m}} \frac{x_{2 m}}{n^{m}}+O\left(\frac{1}{n^{M+1}}\right)\right) .
\end{aligned}
$$

Для обоснования соотношения (6) используем метод перевала, исходя из интегрального представления (3). В рассматриваемой ситуации имеется единственная точка перевала $z_{0}$ функции $\omega$, определяемая равенством $z_{0}=\alpha(1-p) /[p(1-\alpha)]>1$.

Будем интегрировать в (3) по окружности $|z|=z_{0}$ (пробегаемой в положительном направлении). На этой окружности лежит точка перевала $z=z_{0}$ функции $\omega$, для которой $\omega^{\prime}\left(z_{0}\right)=0$ и

$$
\omega^{\prime \prime}\left(z_{0}\right)=\frac{(1-\alpha)^{3} p^{2}}{\alpha(1-p)^{2}}>0 .
$$

Таким образом, точка $z=z_{0}$ является точкой перевала первого порядка этой функции, причем из условий $|z|=z_{0}, z \neq z_{0}$ следует, что

$$
\operatorname{Re} \omega(z)<\omega\left(z_{0}\right)=-\alpha \ln \frac{\alpha}{p}-(1-\alpha) \ln \frac{1-\alpha}{1-p}<0 .
$$

Поскольку функции $\varphi$ и $\omega$ аналитичны в некоторой области (круговом кольще), содержащей контур интегрирования в (3) (с разрезом по отрищательной вещественной полуоси), то при $n \rightarrow \infty$ в условиях теоремы 1 справедливо аскмптотическое разложение (см. [3, с. 66]):

$$
\begin{aligned}
& \frac{1}{2 \pi i} \oint_{|z|=z_{0}>1} \varphi(z) \exp (n \omega(z)) d z \\
& \sim \frac{1}{2 \pi i} \sqrt{\frac{\pi}{n}}\left(\frac{p}{\alpha}\right)^{\alpha n}\left(\frac{1-p}{1-\alpha}\right)^{(1-\alpha) n} \sum_{m=0}^{\infty} \frac{(2 m) !}{m ! 2^{2 m}} \frac{a_{2 m}}{n^{m}} .
\end{aligned}
$$

При этом из условий (1) следует, что разложение (9) имеет место равномерно относительно $\alpha \in\left[\alpha_{0}, \alpha_{1}\right]$. Коэффициенты $a_{m}(m=0,1, \ldots)$ в (9) определяются из соотношений (см. $[3$, с. 66]):

$$
a_{0}=i \varphi\left(z_{0}\right) \sqrt{\frac{2}{\omega^{\prime \prime}\left(z_{0}\right)}}=\frac{i(1-p)}{\alpha-p} \sqrt{\frac{2 \alpha}{1-\alpha}}
$$


(последнее равенство следует из (4) и (5));

$$
\begin{aligned}
\omega\left(z_{0}+\psi(u)\right) & =\omega\left(z_{0}\right)-u^{2} \\
\varphi\left(z_{0}+\psi(u)\right) \psi^{\prime}(u) & =\sum_{m=0}^{\infty} a_{m} u^{m} .
\end{aligned}
$$

При этом полагаем

$$
\psi(0)=0 ; \quad \psi^{\prime}(0)=i \sqrt{\frac{2}{\omega^{\prime \prime}\left(z_{0}\right)}}=\frac{i(1-p)}{p(1-\alpha)} \sqrt{\frac{2 \alpha}{1-\alpha}}
$$

Подставляя значения функций $\varphi$ и $\omega$ и их производных, получаем, что при всех достаточно малых по модулю значениях $u$

$$
\begin{gathered}
\ln \left(\frac{p}{\alpha} z_{0}+p \psi(u)\right)-\alpha \ln \left(z_{0}+\psi(u)\right)+J+u^{2}=0 \\
\frac{\psi^{\prime}(u)}{z_{0}+\psi(u)-1}=\sum_{m=0}^{\infty} a_{m} u^{m}
\end{gathered}
$$

При этом величина $J$ в (10) определена в формулировке теоремы 1. Полагая $x_{m}=a_{m} / a_{0}(m=0,1, \ldots)$, имеем $x_{0}=1$. Из равенства

$$
a_{0}=\frac{i(1-p)}{\alpha-p} \sqrt{\frac{2 \alpha}{1-\alpha}}
$$

получаем, что асимптотическое разложение (9) можно записать в виде (6).

Продифференцировав по переменной $u$ равенство (10), выводим, что функция $\psi$ удовлетворяет дифференциальному уравнению

$$
\alpha(1-\alpha) \psi(u) \psi^{\prime}(u)+2 u\left(z_{0}+\alpha \psi(u)\right)\left(z_{0}+\psi(u)\right)=0
$$

Положим

$$
\psi(u)=\sum_{r=0}^{\infty} c_{r} u^{r}
$$

тогда

$$
c_{0}=\psi(0)=0, \quad c_{1}=\psi^{\prime}(0)=\frac{i(1-p)}{p(1-\alpha)} \sqrt{\frac{2 \alpha}{1-\alpha}}
$$

и

$$
\psi^{\prime}(u)=\sum_{r=0}^{\infty}(r+1) c_{r+1} u^{r}
$$

Подставляя в (12) разложения в степенные ряды функций $\psi$ и $\psi^{\prime}$ и перемножая эти ряды по Коши, получаем, что коэффициенты $c_{r}(r=$ 
$1,2, \ldots)$ удовлетворяют системе нелинейных рекуррентных соотношений (8). Из равенства (12) нетрудно вывести, что

$$
c_{2}=\frac{1}{2} \psi^{\prime \prime}(0)=-\frac{2(1+\alpha)(1-p)}{3 p(1-\alpha)^{2}} .
$$

Записывая (11) в виде

$$
\left(z_{0}-1+\psi(u)\right) \sum_{m=0}^{\infty} a_{m} u^{m}=\sum_{r=0}^{\infty}(r+1) c_{r+1} u^{r}
$$

и перемножая соответствующие степенные ряды, убеждаемся в том, что коэффициенты $a_{m}(m=0,1, \ldots)$ удовлетворяют системе линейных рекуррентных соотношений

$$
a_{m}=\frac{p(1-\alpha)}{\alpha-p}\left((m+1) c_{m+1}-\sum_{r=0}^{m-1} c_{m-r} a_{r}\right) \quad(m=1,2, \ldots)
$$

с начальным условием

$$
a_{0}=\frac{i(1-p)}{\alpha-p} \sqrt{\frac{2 \alpha}{1-\alpha}}
$$

Подставляя в эти соотношения значения $a_{m}=a_{0} x_{m}(m=0,1, \ldots)$, получаем равенства (7) с начальным условием $x_{0}=1$. Теорема 1 доказана.

Следствие 1. При условиях теоремы 1 равномерно относительно $\alpha \in\left[\alpha_{0}, \alpha_{1}\right]$

$$
\begin{aligned}
\mathbf{P}\{\xi \geqslant k\}= & \frac{1-p}{\alpha-p} \sqrt{\frac{\alpha}{2 \pi(1-\alpha) n}} \exp (-n J) \\
& \times\left(1+\frac{1}{12 n}\left(\frac{\alpha^{2}+10 \alpha p+p^{2}-12 p}{(\alpha-p)^{2}}\right.\right. \\
& \left.\left.-\frac{1}{\alpha(1-\alpha)}\right)+O\left(n^{-2}\right)\right)
\end{aligned}
$$

Для обоснования формулы (13) используем равенства (8) при $r=2$. С учетом заданных начальных условий получаем

$$
\begin{aligned}
c_{3} & =\frac{i}{4} \sqrt{\frac{1-\alpha}{2 \alpha}}\left(\frac{2(1+\alpha)}{1-\alpha} c_{2}+\frac{p}{1-p}\left(2 c_{1}^{2}+2(1-\alpha) c_{2}^{2}\right)\right) \\
& =-\frac{i(1-p)\left(\alpha^{2}+11 \alpha+1\right)}{9 p \sqrt{2 \alpha(1-\alpha)^{5}}}
\end{aligned}
$$


Используя равенства (7) при $m=1$ и $m=2$ и учитывая начальное условие $x_{0}=1$, последовательно получаем:

$$
\begin{aligned}
& x_{1}=-\frac{2 i(\alpha(1-p-2 \alpha)+2 p)}{3(\alpha-p) \sqrt{2 \alpha(1-\alpha)}} \\
& x_{2}=\frac{\alpha^{2}+10 \alpha p+p^{2}-12 p}{6(\alpha-p)^{2}}-\frac{1}{6 \alpha(1-\alpha)} .
\end{aligned}
$$

Теперь равенство (13) следует из (6), если в соответствуюшем разложении ограничиться первыми двумя слагаемыми с остаточным членом порядка $O\left(n^{-2}\right)$.

Заметим, что формула (13) может быть получена также из равенства $x_{2}=a_{2} / a_{0}$ и соотношения (см. $[4$, с. 165]):

$$
\begin{aligned}
a_{2}=-\frac{i}{\sqrt{2\left(\omega^{\prime \prime}\left(z_{0}\right)\right)^{3}}} & \left(2 \varphi^{\prime \prime}\left(z_{0}\right)-\frac{2 \omega^{\prime \prime \prime}\left(z_{0}\right) \varphi^{\prime}\left(z_{0}\right)}{\omega^{\prime \prime}\left(z_{0}\right)}\right. \\
& \left.+\varphi\left(z_{0}\right)\left(\frac{5\left(\omega^{\prime \prime \prime}\left(z_{0}\right)\right)^{2}}{6\left(\omega^{\prime \prime}\left(z_{0}\right)\right)^{2}}-\frac{\omega^{(\text {iv })}\left(z_{0}\right)}{2 \omega^{\prime \prime}\left(z_{0}\right)}\right)\right) .
\end{aligned}
$$

Следствие 2. При условиях теоремы 1 равномерно относительно $\alpha \in\left[\alpha_{0}, \alpha_{1}\right]$

$$
\mathbf{P}\{\xi \geqslant k\}=C_{n}^{k} p^{k}(1-p)^{n-k} \frac{\alpha(1-p)}{\alpha-p}\left(1-\frac{p(1-\alpha)}{(\alpha-p)^{2} n}+O\left(n^{-2}\right)\right) .
$$

Равенство (14) следует из (13) по формуле Стирлинга.

Следствие 3. Пусть при $n \rightarrow \infty$ и постоянном $p \in(0,1) k \rightarrow \infty$ $(k \in \mathbf{N}) \max$, чmo $\lim _{n \rightarrow \infty}(k / n)=\beta \in(p, 1)$.

Tozda

$$
0<\lim _{n \rightarrow \infty} \frac{\mathbf{P}\{\xi=k\}}{\mathbf{P}\{\xi \geqslant k\}}=\frac{\beta-p}{\beta(1-p)}<1 .
$$

Заметим, что утверждение следствия 3 легко выводится из результатов работы [1]. Более общее утверждение для случая полиномиального распределения приведено в [2].

Teорема 2. Пусть $n p u n \rightarrow \infty 0<p=$ const $<1 u k \rightarrow \infty(k \in \mathbf{N})$ так, что $0<\alpha_{0} \leqslant \alpha=k / n \leqslant \alpha_{1}<p\left(\alpha_{0}, \alpha_{1}-\right.$ постоянные $)$. Тогда равномерно относительно $\alpha \in\left[\alpha_{0}, \alpha_{1}\right]$ справедливо асимптотическое разложение

$$
\mathbf{P}\{\xi \leqslant k\} \backsim \frac{p}{p-\alpha} \sqrt{\frac{1-\alpha}{2 \pi \alpha n}} \exp (-n J)\left(1+\sum_{m=1}^{\infty} \frac{(2 m) !}{m ! 2^{2 m}} \frac{y_{2 m}}{n^{m}}\right),
$$

где коэффициенты $y_{m}(m=1,2, \ldots)$ определяются из соотношений (7) $u$ (8) и соответствующих начальных условий с заменой $\alpha$ на $1-\alpha, p$ на $1-p$ и $x_{m}$ на $y_{m}$. 
Для обоснования формулы (15) достаточно заметить, что с учетом произведенной замены она следует из (6).

Следствие 4. Пусть при $n \rightarrow \infty$ и постоянном $p \in(0,1) k \rightarrow \infty$ $(k \in \mathbf{N})$ maк, umo $\lim _{n \rightarrow \infty}(k / n)=\beta \in(0, p)$.

Тогда

$$
0<\lim _{n \rightarrow \infty} \frac{\mathbf{P}\{\xi=k\}}{\mathbf{P}\{\xi \leqslant k\}}=\frac{p-\beta}{p(1-\beta)}<1 .
$$

Рассмотрим теперь задачу оценки вероятности $\mathbf{P}\{\eta \geqslant k\}$ при условии (2). Для получения разложения этой вероятности в асимптотический степенной ряд по степеням переменной $\lambda^{-1}$ будем исходить из равенств

$$
\mathbf{P}\{\eta=n\}=\frac{\lambda^{n}}{n !} e^{-\lambda}=\frac{1}{2 \pi i} \oint \frac{e^{\lambda(z-1)}}{z^{n+1}} d z \quad(n=0,1, \ldots) .
$$

Интегрирование в формуле (16) осупествляется по окружности с центром в нуле, пробегаемой в положительном направлении. Далее будем считать, что $R>1$, где $R-$ радиус указанной окружности. Если $k \in \mathbf{N}$, то из условия $|z|=R>1$ следует, что

$$
\mathbf{P}\{\eta \geqslant k\}=\sum_{n=k}^{\infty} \mathbf{P}\{\eta=n\}=\frac{1}{2 \pi i} \oint \varphi(z) \exp \left(\lambda \omega_{0}(z)\right) d z,
$$

где функция $\varphi$ определяется из (4) и

$$
\omega_{0}(z)=z-1-\frac{k}{\lambda} \ln z=z-1-\gamma \ln z
$$

если выполнено условие (2).

Теорема 3. Пусть $\lambda \rightarrow+\infty, k \rightarrow \infty(k \in \mathbf{N})$ так, ито выполнено условие (2). Тогда равномерно относительно $\gamma \in\left[\gamma_{0}, \gamma_{1}\right]$ справедливо асимптотическое разложение

$$
\begin{aligned}
\mathbf{P}\{\eta \geqslant k\} \backsim & \frac{1}{\gamma-1} \sqrt{\frac{\gamma}{2 \pi \lambda}} \exp \left(-\lambda \psi_{0}(\gamma)\right) \\
& \times\left(1+\sum_{m=1}^{\infty} \frac{(2 m) !}{m ! 2^{2 m}} \frac{v_{2 m}}{\lambda^{m}}\right),
\end{aligned}
$$

где $\psi_{0}(\gamma)=1-\gamma+\gamma \ln \gamma>0$ и коэффичиенты $v_{m}(m=1,2, \ldots)$ удовлетворяют линейным рекуррентным соотношениям

$$
v_{m}=\frac{m+1}{i \sqrt{2 \gamma}} d_{m+1}-\frac{1}{\gamma-1} \sum_{r=0}^{m-1} d_{m-r} v_{r} \quad(m=1,2, \ldots)
$$

с начальным условием $v_{0}=1$. В формуле $(20)$ коэффичиенты $d_{m}(m=$ 
$1,2, \ldots)$ удовлетворяют нелинейньм рекуррентным соотночениям

$$
d_{m}=\frac{i}{(m+1) \sqrt{2 \gamma}}\left(2 d_{m-1}+\sum_{r=2}^{m-1}(m-r+1) d_{r} d_{m-r+1}\right) \quad(m=3,4, \ldots)
$$

с начальными условиями

$$
d_{1}=i \sqrt{2 \gamma}, \quad d_{2}=-\frac{2}{3}
$$

Д ока зате ль с т в о. Используем метод перевала, учитывая интегральное представление (17). Точки перевала функции $\omega_{0}$ удовлетворяют уравнению $\omega_{0}^{\prime}(z)=0$. Из этого уравнения находим: $z=\gamma>1$.

Будем интегрировать в (17) по окружности $|z|=\gamma$ (пробегаемой в положительном направлении). На этой окружности имеется единственная точка перевала $z=\gamma$ функции $\omega_{0}$, для которой $\omega_{0}^{\prime}(\gamma)=0$ и $\omega_{0}^{\prime \prime}(\gamma)=1 / . \gamma>0$. Таким образом, точка $z=\gamma$ является точкой перевала первого порядка этой функции, причем из условий $|z|=\gamma, z \neq \gamma$ следует, что

$$
\operatorname{Re} \omega_{0}(z)<\omega_{0}(\gamma)=\gamma-1-\gamma \ln \gamma
$$

(заметим, что в (18) выбирается та непрерывная ветвь логарифма, которая принимает вещественные значения на вещественной положительной полуоси). Поскольку функции $\varphi$ и $\omega_{0}$ аналитичны в некоторой области (круговом кольце), содержащей контур интегрирования в (17) (с разрезом по отрицательной вещественной полуоси), то при $\lambda \rightarrow+\infty$ (см. [1, c. 66]) справедливо асимптотическое разложение:

$$
\begin{aligned}
& \frac{1}{2 \pi i} \oint \varphi(z) \exp \left(\lambda \omega_{0}(z)\right) d z \\
& \backsim \frac{1}{2 \pi i} \sqrt{\frac{\pi}{\lambda}} \exp \left(\lambda \omega_{0}(\gamma)\right) \sum_{m=0}^{\infty} \frac{(2 m) !}{m ! 2^{2 m}} \frac{b_{2 m}}{\lambda^{m}} .
\end{aligned}
$$

При этом $\omega_{0}(\gamma)=-\psi_{0}(\gamma)$, где функция $\psi_{0}$ определена в условии теоремы 1. Кроме того, из (2) следует, что разложение (22) имеет место равномерно относительно $\gamma \in\left[\gamma_{0}, \gamma_{1}\right]$. Коэффициенты $b_{m}(m=0,1, \ldots)$ в (22) определяются из соотношений (см. $[1$, с. 66]):

$$
b_{0}=i \varphi(\gamma) \sqrt{\frac{2}{\omega_{0}^{\prime \prime}(\gamma)}}=\frac{i \sqrt{2 \gamma}}{\gamma-1}
$$

(последнее равенство следует из (4));

$$
\begin{aligned}
\omega_{0}(\gamma+h(u)) & =\omega_{0}(\gamma)-u^{2} \\
\varphi_{0}(\gamma+h(u)) h^{\prime}(u) & =\sum_{m=0}^{\infty} b_{m} u^{m}
\end{aligned}
$$

3 Теория вероятностей и ее применения, № 1 
При этом

$$
h(0)=0, \quad h^{\prime}(0)=i \sqrt{\frac{2}{\omega_{0}^{\prime \prime}(\gamma)}} .
$$

Подставляя значения функций $\varphi$ и $\omega_{0}$ и их производных, получаем, что при всех достаточно малых по модулю значениях $u$

$$
\begin{gathered}
\gamma \ln \left(1+\frac{h(u)}{\gamma}\right)=h(u)+u^{2} \\
\frac{h^{\prime}(u)}{\gamma+h(u)-1}=\sum_{m=0}^{\infty} b_{m} u^{m} \\
h(0)=0 ; \quad h^{\prime}(0)=i \sqrt{2 \gamma}
\end{gathered}
$$

Полагая $v_{m}=b_{m} / b_{0}(m=0,1, \ldots)$, имеем $v_{0}=1$. Из равенства $b_{0}=i \sqrt{2 \gamma} /(\gamma-1)$ получаем, что асимптотическое разложение (22) можно записать в виде (19).

Продифференцировав по переменной $u$ равенство (23), выводим, что функция $h$ удовлетворяет дифференциальному уравнению

$$
h(u)\left(h^{\prime}(u)+2 \dot{u}\right)+2 \gamma u=0 .
$$

Положим $h(u)=\sum_{m=0}^{\infty} d_{m} u^{m}$, тогда

$$
\begin{aligned}
d_{0} & =h(0)=0, \quad d_{1}=h^{\prime}(0)=i \sqrt{2 \gamma}, \\
h^{\prime}(u) & =\sum_{m=0}^{\infty}(m+1) d_{m+1} u^{m} .
\end{aligned}
$$

Подставляя в (25) разложения в степенные ряды функций $h$ и $h^{\prime}$ и перемножая эти ряцы по Коши, получаем, что коэффициенты $d_{m}$ $(m=1,2, \ldots)$ удовлетворяют системе нелинейных рекуррентных соотношений (21). Из равенства (25) кетрудно выпести, что $d_{2}=\frac{1}{2} h^{\prime \prime}(0)=-\frac{2}{3}$.

Записывая (24) в виде

$$
(\gamma-1+h(u)) \sum_{m=0}^{\infty} b_{m} u^{m}=h^{\prime}(u)=\sum_{m=0}^{\infty}(m+1) d_{m+1} u^{m}
$$

и перемножая соответствуюшие степенные ряды, убеждаемся в том, что коэффициенты $b_{m}(m=0,1, \ldots)$ удовлетворяют системе линейных рекуррентных соотношений

$$
b_{m}=\frac{1}{\gamma-1}\left((m+1) d_{m+1}-\sum_{r=0}^{m-1} d_{m-r} b_{r}\right) \quad(m=1,2, \ldots)
$$

с начальным условием $b_{0}=i \sqrt{2 \gamma} /(\gamma-1)$. Подставляя в эти соотношения $b_{m}=b_{0} v_{m}(m=0,1, \ldots)$, получаем равенства $(20)$ с начальным условием $v_{0}=1$. Теорема 3 доказана. 
Следствие 5. При условиях теоремы 3

$$
\begin{aligned}
\mathbf{P}\{\eta \geqslant k\}= & \frac{1}{\gamma-1} \sqrt{\frac{\gamma}{2 \pi \lambda}} \exp \left(-\lambda \psi_{0}(\gamma)\right) \\
& \times\left(1-\frac{\gamma^{2}+10 \gamma+1}{12 \gamma(\gamma-1)^{2}} \lambda^{-1}\right. \\
& \left.\quad+\frac{\gamma^{4}+308 \gamma^{3}+534 \gamma^{2}+20 \gamma+1}{288 \gamma^{2}(\gamma-1)^{4}} \lambda^{-2}+O\left(\lambda^{-3}\right)\right)
\end{aligned}
$$

Для обоснования формулы (26) используем равенства (21). С учетом заданных начальных условий путем элементарных выкладок последовательно получаем:

$$
d_{3}=-\frac{i}{9 \sqrt{2 \gamma}}, \quad d_{4}=-\frac{2}{135 \gamma}, \quad d_{5}=\frac{i}{540 \gamma \sqrt{2 \gamma}} .
$$

Используя теперь равенства (20) с начальным условием $v_{0}=1$, получаем:

$$
\begin{aligned}
& v_{1}=-\frac{2(\gamma+2) i}{3(\gamma-1) \sqrt{2 \gamma}} \\
& v_{2}=-\frac{\gamma^{2}+10 \gamma+1}{6 \gamma(\gamma-1)^{2}} \\
& v_{3}=\frac{i\left(8 \gamma^{3}+336 \gamma^{2}+204 \gamma-8\right)}{135 \gamma(\gamma-1)^{3} \sqrt{2 \gamma}} \\
& v_{4}=\frac{\gamma^{4}+308 \gamma^{3}+534 \gamma^{2}+20 \gamma+1}{216 \gamma^{2}(\gamma-1)^{4}} .
\end{aligned}
$$

Теперь равенство (26) следует из (19), если в соответствующем разложении ограничиться первыми тремя слагаемыми с остаточным членом порядка $O\left(\lambda^{-3}\right)$.

Следствие 6. При условиях теоремы 3

$$
\begin{aligned}
\mathbf{P}\{\eta \geqslant k\}= & \frac{\lambda^{k}}{k !} e^{-\lambda} \frac{\gamma}{\gamma-1} \\
& \times\left(1-\frac{1}{(\gamma-1)^{2}} \lambda^{-1}+\frac{\gamma+2}{(\gamma-1)^{4}} \lambda^{-2}+O\left(\lambda^{-3}\right)\right) .
\end{aligned}
$$

Формула (27) следует из (26), если учесть, что в условиях теоремы 3 (cM. $[5$, c. 80$])$ :

$$
\begin{aligned}
k != & (\gamma \lambda) !=\sqrt{2 \pi \gamma \lambda} \gamma^{\gamma \lambda} \lambda^{\gamma \lambda} \exp (-\gamma \lambda) \\
& \times\left(1+\frac{1}{12 \gamma} \lambda^{-1}+\frac{1}{288 \gamma^{2}} \lambda^{-2}+O\left(\lambda^{-3}\right)\right) .
\end{aligned}
$$


Следствие 7. Пусть $k \rightarrow \infty$ так, ито

$$
\lim _{\lambda \rightarrow+\infty} \frac{k}{\lambda}=\delta>1 \quad(k \in \mathbf{N}) .
$$

Toгda

$$
0<\lim _{\lambda \rightarrow+\infty} \frac{\mathbf{P}\{\eta=k\}}{\mathbf{P}\{\eta \geqslant k\}}=1-\frac{1}{\delta}<1 .
$$

\section{СПИСОК ЛИТЕРАТУРЫ}

1. Санов И. Н. О вероятности больших отклонений случайных величин. - Матем. сб., 1957 , т. 42 , в. 1 , с. 11-44.

2. Тимашев $A$. H. Теоремы о больших уклонениях в полиномиальной схеме испытаний. - Дискретн. матем., 1993, т. 5, в. 2, с. 83-89.

3. Евграфов М. А. Асимптотические оценки и целые функции. М.: Физматлит, $1962,200 \mathrm{c}$.

4. Олвер $Ф$. Введение в асимптотические методы и специальные функции. М.: Наука, $1978,376 \mathrm{c}$.

5. Копсон Э. Асимптотические разложения. М.: Мир, 1966, 159 с.

Поступила в редакцию 20.XII.1996

Исправленный вариант 10.VII.1997 